\title{
OPEN Prognostic significance of p53, Sox11, and Pax5 co-expression in mantle cell lymphoma
}

\author{
Caixia Jing ${ }^{1,2,4}$, Yuhuan Zheng ${ }^{1,3,4}$, Yu Feng ${ }^{1}$, Xia Cao ${ }^{1}$ \& Caigang Xu
}

Mantle cell lymphoma (MCL) is a relatively rare subtype of non-Hodgkin's lymphoma. To identify molecular biomarkers in $\mathrm{MCL}$, we performed immunohistochemistry tissue arrays using biopsies from 64 MCL patients diagnosed in West China Hospital from 2012 to 2016. TP53 mutation status in those patients was also examined by sequencing. The sequencing results showed TP53 mutations were highly heterogeneous in MCL. We identified four novel TP53 mutations in MCL: P151R, G199R, $\mathrm{V} 218 \mathrm{E}$, and G325R. The MCL patients with TP53 mutations had inferior progression-free survival (PFS, $p=0.002$ ) and overall survival $(O S, p=0.011)$. Tissue array results showed the expression of $p 53$, Sox11, or Pax5 alone did not correlate with the patient PFS and OS. However, the MCL patients with triple-positive expression of $p 53 /$ Sox11/Pax5 had inferior PFS $(p=0.008)$ and $O S(p=0.002)$. Such risk stratification was independent to the mantle cell lymphoma international prognostic index (MIPI), $\mathrm{Ki}-67$ value, and TP53 mutation status of the patients. The triple-positive patients might represent a subtype of high-risk MCL. Our findings might indicate a novel way to stratify MCL and predict patients' prognosis.

Mantle cell lymphoma (MCL) is a mature B-cell non-Hodgkin's lymphoma (NHL) and accounts for about $6 \%$ of all NHL cases ${ }^{1}$. Based on an epidemiology study in the United States, the incidence rate of MCL was about 0.5 to 0.6 per 100,000 persons, and the rate has increased 2-3 times in the past decades ${ }^{2}$. The primary genetic event of MCL is translocation $\mathrm{t}(11 ; 14)(\mathrm{q} 13 ; \mathrm{q} 32)$, which results in cyclin D1 overexpression. However, the dysregulation of cyclin D1 alone may not be sufficient to trigger MCL pathogenesis and aggressiveness ${ }^{3}$. There are many heterogeneous secondary genetic alterations in MCL. For examples, mutations in $I N K 4 A^{4}, A T M^{5}, C D K 4^{6}$, and $T P 53^{7}$ genes are commonly seen in different MCL patients. Those alterations further target the cell signaling pathways, such as cell cycle progression, DNA damage response, and cell survival regulation, thus promoting tumor malignancy ${ }^{3,8}$. MCL remains an incurable lymphoma without any standardized first-line treatment strategy. In addition, MCL is a highly heterogeneous disease; some MCL is aggressive with a median survival of only 3 years while some MCL is indolent that patients can be observed for a period of time before initiating their first treatment ${ }^{9,10}$. Identification of prognostic biomarkers in MCL provides insight for MCL research, not only for risk stratification and personalized treatment optimization but also for demonstrations of MCL biology. In this study, we examined p53, Sox11, and Pax5 expressions in MCL prognosis. Our data suggested a novel risk stratification of MCL based on immunohistochemistry (IHC) analysis of target antigens' expression.

\section{Results}

TP53 mutations in mantle cell lymphoma. To investigate TP53 mutations in MCL, we used 64 patient tissue biopsies for target gene sequencing. The patients' characteristics at diagnosis are summarized in Table 1 and Fig. 1. We found 12 TP53 mutations in 11 MCL patients (17.19\%), and one patient had 2 TP53 mutations (Supplementary Fig. S1, Fig. 2). There were 10 missense mutations (83.33\%), one nonsense mutation (8.33\%), and one frame-shift mutation (8.33\%). Five TP53 mutations occurred in exon 5, three mutations in exon 8 , two mutations in exon 6, and one mutation in exon 7 and 9, respectively. Eleven mutations resulted in amino acid alteration in the p53 DNA binding domain (91.67\%), while one resulted in amino acid alteration at the C terminal of $\mathrm{p} 53$.

\footnotetext{
${ }^{1}$ Department of Hematology/Hematology Research Laboratory, West China Hospital, Sichuan University, \#37 Guo Xue Xiang Street, Chengdu 610041, China. ${ }^{2}$ Department of Hematology, Affiliated Hospital of Southwest Medical University, Luzhou, China. ${ }^{3}$ State Key Laboratory of Biotherapy and Cancer Centre, West China Hospital, Sichuan University, Chengdu, China. ${ }^{4}$ These authors contributed equally: Caixia Jing and Yuhuan Zheng. ${ }^{\square}$ email: xucaigang@wchscu.cn
} 


\begin{tabular}{|l|l|}
\hline & Total Pt \# 64 \\
\hline Median age (range) & $61(36-82)$ \\
\hline Male, no. (\%) & $52(81.3)$ \\
\hline HB, g/L (mean \pm SD) & $122.62 \pm 23.82$ \\
\hline Median WBC, 10\%/L (range) & $7.25(2.97-70.21)$ \\
\hline Median PLT, 10/L (range) & $137(38-407)$ \\
\hline Median LDH, IU/L (range) & $220(136-827)$ \\
\hline MIPI ${ }^{\text {high }}$, no. (\%) & $14 / 56(21.9)$ \\
\hline Median Ki-67, \% (range) & $25(4-80)$ \\
\hline CD5-, no. (\%) & $10(15.6)$ \\
\hline Morphological variants & \\
\hline Classical, no. (\%) & $40 / 61(65.6)$ \\
\hline Pleomorphic, no. (\%) & $6 / 61(9.8)$ \\
\hline Blastoid variant, No. (\%) & $15 / 61(24.6)$ \\
\hline Treatment & $18(28.1)$ \\
\hline CHOP (\%) & $40(62.5)$ \\
\hline R-CHOP (\%) & $3(4.7)$ \\
\hline R-CHOP/DHAP (\%) & $1(1.6)$ \\
\hline R-CHOP/R maintenance (\%) & $1(1.6)$ \\
\hline R-CHOP/DHAP/R maintenance (\%) & $1(1.6)$ \\
\hline R-CHOP/DHAP/ASCT (\%) & \\
\hline
\end{tabular}

Table 1. Mantle cell lymphoma patients' characteristics. $H B$ hemoglobin, $S D$ standard deviation, $W B C$ white blood cell, $L D H$ lactate dehydrogenase, MIPI mantle cell lymphoma international prognostic index.

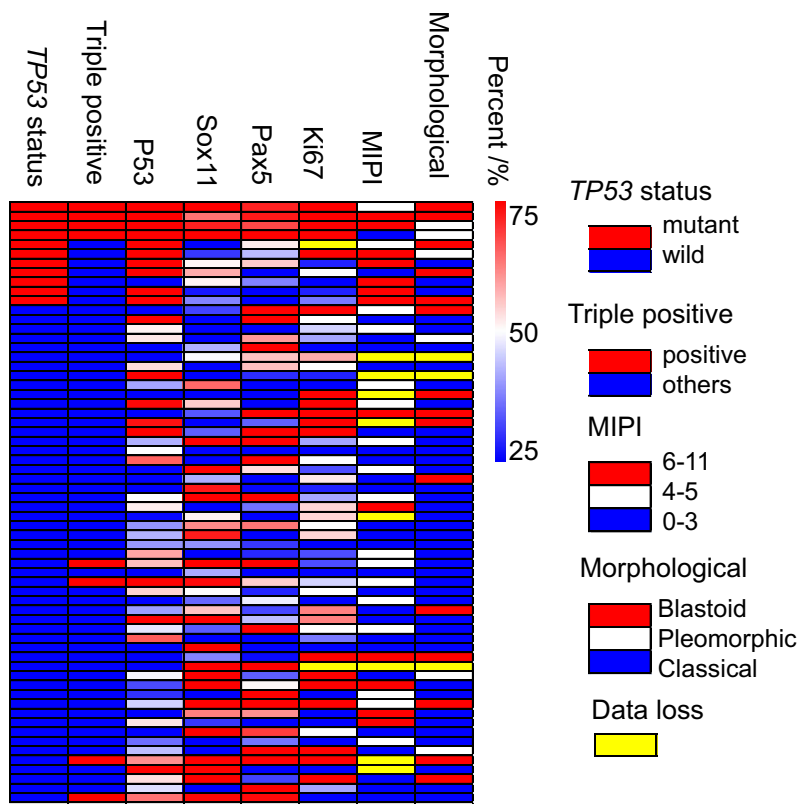

Figure 1. Heatmap of mantle cell lymphoma patients' characteristics.

We reviewed literatures and databases for identified TP53 mutations in MCL (Supplementary Table S1). In 18 independent studies of a total of 956 MCL patients, 151 patients had TP53 mutations (15.8\%). TP53 mutations in MCL exhibited high heterogeneity, located at more than 70 different sites in the TP53 gene. Even the most frequently mutated amino acid residue of p53, R248, occurred only in 13 MCL patients (8.61\% in all 151 TP53 mutated MCL patients). In addition, we didn't found significant difference between expression of Sox11 and Pax5 in MCL patients with mutant TP53 and non-mutant TP53.

p53, Sox11, and Pax5 triple-positive mantle cell lymphoma patients have adverse prognosis. As described below, IHC tissue arrays of $64 \mathrm{MCL}$ patient's biopsy samples had been performed and representative staining was shown in Fig. 3a-c. The percentage of MCL cells in the biopsy samples and p53 positive 


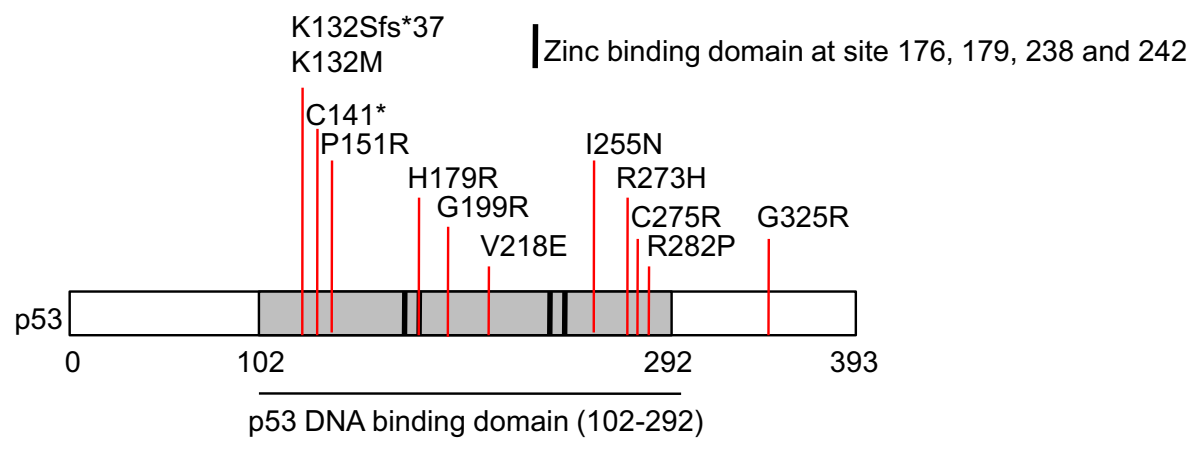

Figure 2. TP53 mutations in West China Hospital cohort of mantle cell lymphoma.

a)

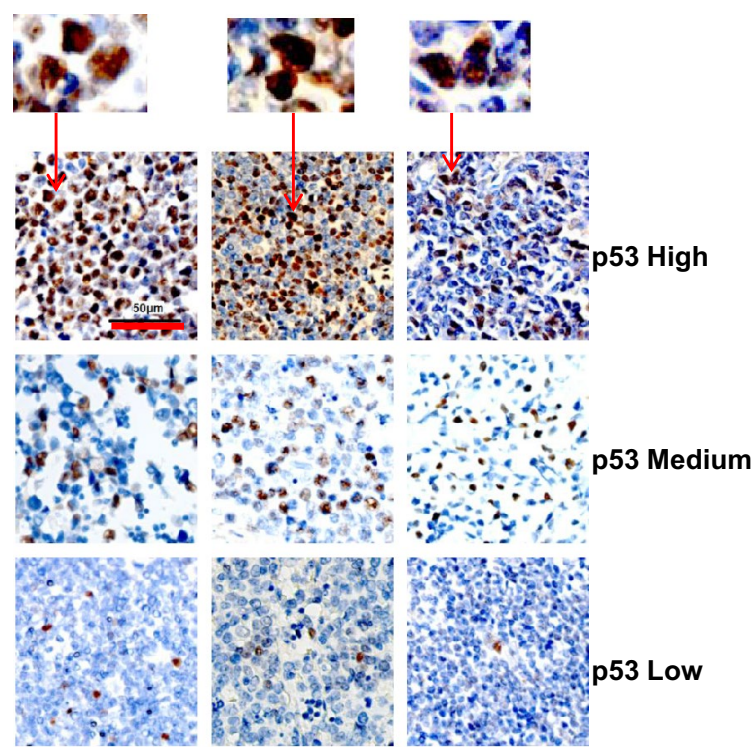

C)

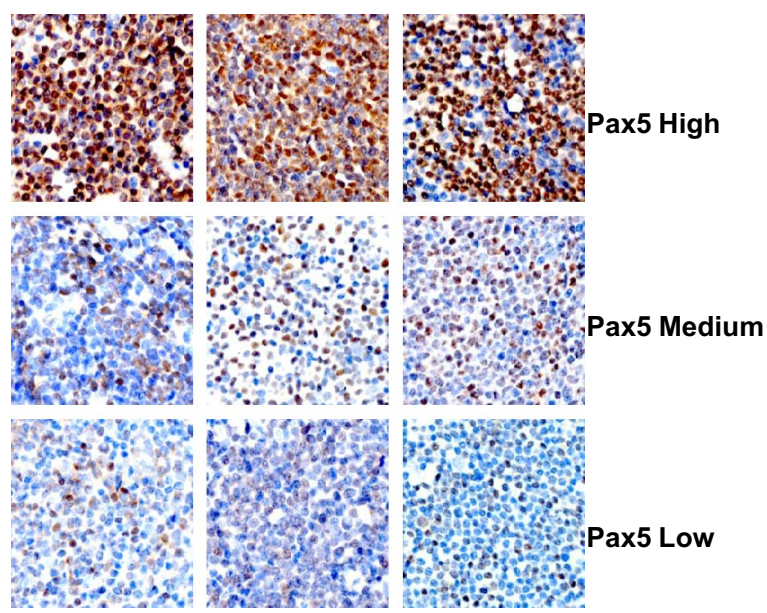

b)

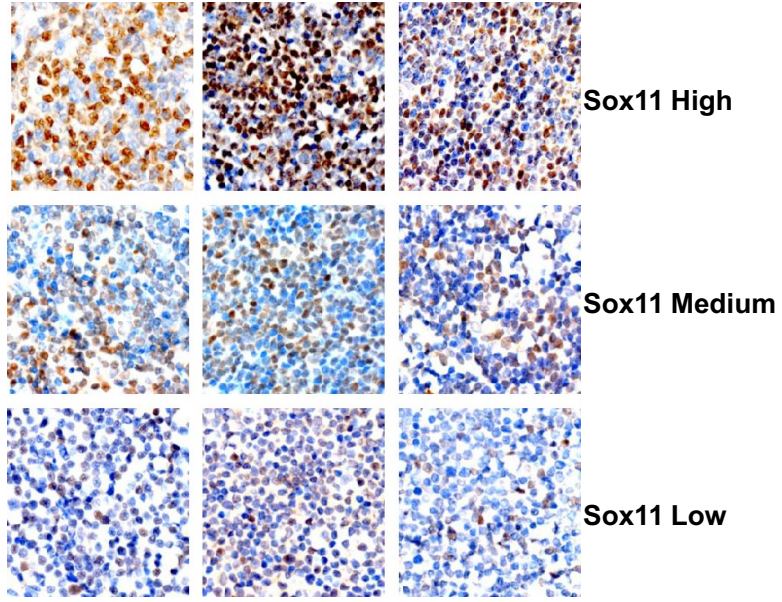

d)

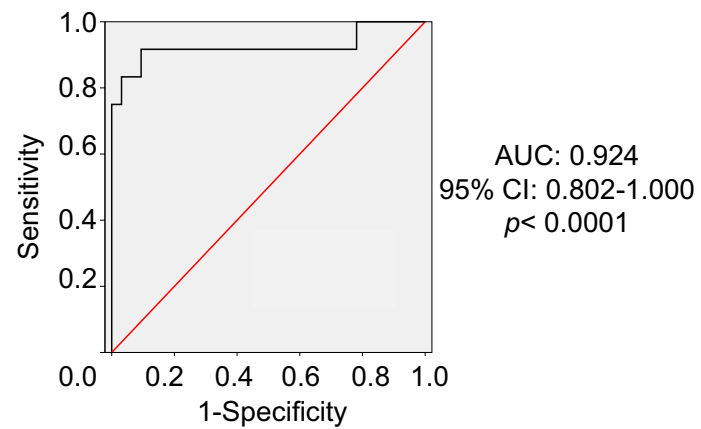

Figure 3. Tissue array of mantle cell lymphoma. Immunohistochemistry tissue arrays of MCL patients' samples for (a) p53, (b) Sox11, and (c) Pax5. Nine representative out of 64 of each protein stainings were presented. The red arrow represented p53 positive cells in the MCL cells and zoomed images were above; (d) ROC curve analysis of p53 immunohistochemistry as a parameter to discriminate between TP53 mutated and non-mutated samples. 


\begin{tabular}{|l|l|l|l|l|l|}
\hline & Negative (\%) & Low positive (\%) & Positive (\%) & High positive (\%) & Total positive (\%) \\
\hline p53 & $42(65.6)$ & $16(25)$ & $3(4.7)$ & $3(4.7)$ & $22(34.4)$ \\
\hline Sox11 & $8(12.5)$ & $25(39.1)$ & $27(42.2)$ & $4(6.3)$ & $56(87.5)$ \\
\hline Pax5 & $3(4.7)$ & $28(43.8)$ & $22(34.4)$ & $11(17.2)$ & $61(95.3)$ \\
\hline
\end{tabular}

Table 2. Result of immunohistochemistry staining.

cells in the MCL cells was shown in Supplementary Table S2. Most (84.4\%) of biopsy samples contained at least $70 \%$ MCL cells. As shown in Table 2, 3 out of 64 samples had high p53 expression, 3 had medium p53 expression, and 16 had low p53 expression. 4 samples had high Sox11 expression, 27 had medium expression, and 25 had low expression. 11 samples had high Pax 5 expression, 22 had medium expression, and 28 had low expression. Next, we quantified our result. The $\mathrm{H}$-scores of each protein expression were calculated as described in Methods and Materials, as well as in Supplementary Fig. S2. The ROC curve showed that high p53 expression (large H-score in p53 IHC) predicted TP53 gene mutation well in the patient (Fig. 3d; cutoff H-score value 62, $95 \%$ confidence interval (CI) $0.802-1.000, p<0.0001$ ). The specificity and sensitivity of p53 IHC in predicting TP53 gene mutation were $89.1 \%$ and $91.7 \%$, respectively. The MCL patients with TP53 mutation (17.19\%) had inferior PFS and OS compared with other patients (Fig. 4a). In addition, the intermediate/high value of Mantle Cell Lymphoma International Prognostic Index (MIPI) (MIPI $\geq 4$ ), high Ki-67 value (Ki-67 > 30\%), and blastoid variant were each correlated with inferior PFS and OS of MCL (Fig. 4b-d, respectively).

Our findings suggested that Sox11 and Pax5 alone do not correlate with MCL PFS and OS (data not shown). In our study cohort, the MCL patients with high p53 expression seemed to have inferior OS, but not statistically significant (Supplementary Fig. S3). Those three proteins, Sox11, Pax5 and p53, randomly expressed in each MCL patients without any obvious co-expressions (Supplementary Fig. S4a). However, further analysis suggested that the MCL patients with triple-positive (or triple-high) expression (H-scores p53>40, Sox11>100, and Pax5 $>100$ ) of target proteins had significantly inferior PFS and OS (Fig. 4e). Double-positive expression of either 2 genes did not correlate with prognosis (data not shown). It was notable that the clinical features of the triplepositive patients and the rest of patients had no statistical difference (Table 3). We summarized TP53 mutation status in triple positive patients versus the others (Supplementary Table S3). We didn't identify any correlations. Some of the features such as MIPI ${ }^{\text {high }}$, Ki- $67^{\text {high }}$, and Blastoid variant were correlated with MCL prognosis, but those features had no enrichment in triple-positive patients. Therefore, the triple-positive stratification of MCL might be independent to those known prognostic factors. Multivariate Cox-regression analysis confirmed that triple-positive stratification was independent to prognostic factors such as TP53 mutation status, Ki-67 value and MIPI (Table 4). In addition, evidence suggested that Sox 11 regulated Pax 5 expression in MCL ${ }^{11}$. However, our IHC array showed that a significant amount of MCL patients didn't have Sox11 and Pax5 co-expression (Supplementary Fig. S4b).

\section{Discussion}

According to the literatures and databases, four TP53 mutations detected in our patients-P151R, G199R, V218E, and G325R - had never been reported in MCL. Among those mutations, P151R, G199R, and V218E were located in the p53 DNA binding domain. Thus, those mutations might affect p53 function and promote oncogenic transformation. G325R mutation had been reported in breast cancer with uncertain clinical significance ${ }^{12}$. This mutation located in the oligomerization domain of p53 and therefore might affect p53 tetramerization ${ }^{13}$. Those mutations expanded the mutation spectrum of TP53 in MCL. Some researchers guessed that the controversial relationship between Sox 11 expression and prognosis of MCL might be influenced by TP53 mutant status ${ }^{14,15}$, but no correlation was found between Sox 11 expression and TP53 mutant status in our study.

MIPI and Ki-67 have been used clinically for MCL-risk stratification and prognosis prediction ${ }^{16}$. Several other factors, such as patients' beta-2 microglobulin level, TP53 mutation status, expressions of Sox11, SOC3, Myc, or Pax5 might also harbor prognostic values in $\mathrm{MCL}^{11,17-20}$. In our study, the prognostic significance of MIPI, Ki-67, and TP53 mutation status in MCL patients was in agreement with previous research results. Those findings in our patient cohort provided confidence of our sample collection. We also investigated prognostic significance of p53, Sox 11, and Pax 5 expression in MCL patients. Previous studies on those 3 factors were controversial. Some studies showed that each of these 3 factors was correlated with MCL prognosis, but others had negative findings ${ }^{20-22}$. In particular, according to the revised 4th edition of "WHO Classification of Tumours of Haematopoietic and Lymphoid Tissues", the non-nodal MCL, which was indolent, had negative Sox 11 expression, therefore Sox $11^{\text {high }}$ MCL patients might have inferior OS in a cohort with both non-nodal and classic MCL patients ${ }^{9,23,24}$. However, Nygren L et al. presented an opposite finding that most indolent MCLs were Sox 11 positive ${ }^{14}$. More recently, an European immunohistochemistry study of MCL biopsies suggested that Sox $11^{\text {low }}$ or p5 $3^{\text {high }}$ patients had inferior $\mathrm{OS}^{15}$. By analyzing peripheral blood, adenopathy, and Sox11 expression et al. synthetically, there was no non-nodal type of MCL in our 64-patient cohort. Based on our results in MCL IHC, neither p53, Sox11, nor Pax5 expression alone was correlated with the patient's PFS or OS. The $\mathrm{p} 53^{\text {high }}$ MCL patients had slightly worse OS than the $\mathrm{p} 53^{\text {low }}$ patients but had no statistical significance. If TP53 ${ }^{\mathrm{mut}}$ patients were excluded in comparison (most TP53 ${ }^{\text {mut }}$ patients had high $\mathrm{p} 53$ expression in IHC), the OS of $\mathrm{p} 53^{\text {high }}$ patients had no difference from other patients. According to previous publications, whether the p53 protein level correlated with MCL OS is still controversial. Several publications suggested that the expression of p53 associated with MCL OS ${ }^{14,15,25}$. However, Greiner et al. assessed p53 expression at the protein level in MCL patients, but only reported correlation between 
a)

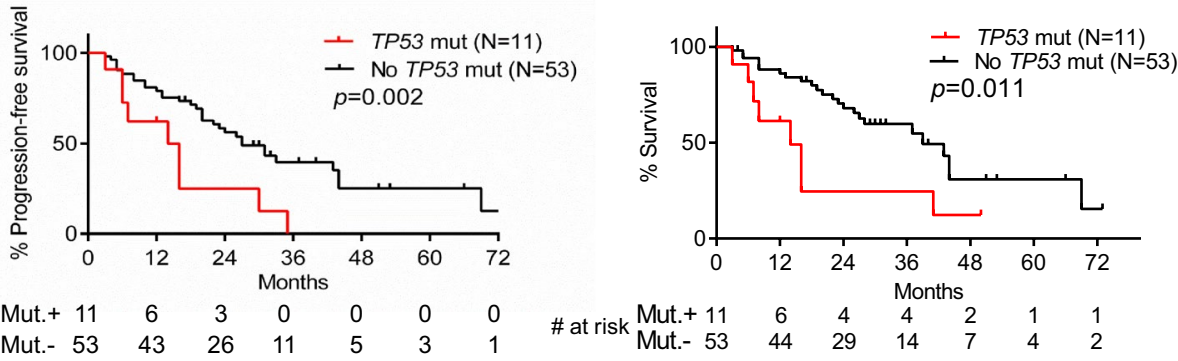

b)
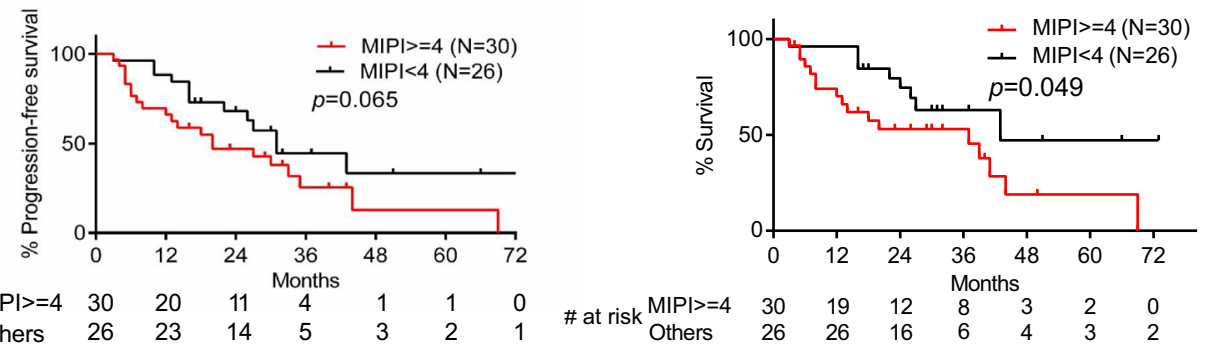

c)

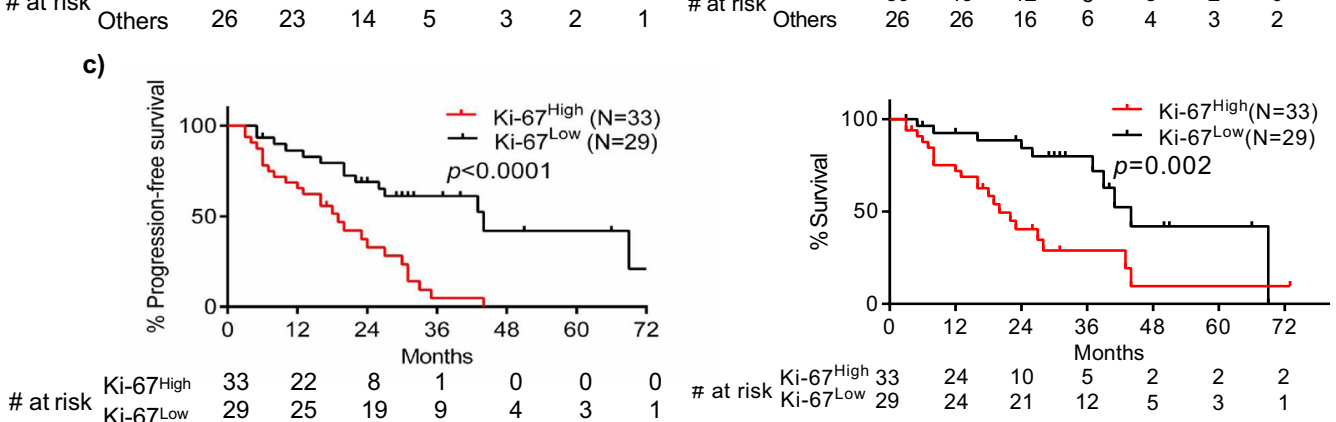

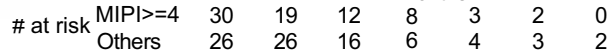

d)

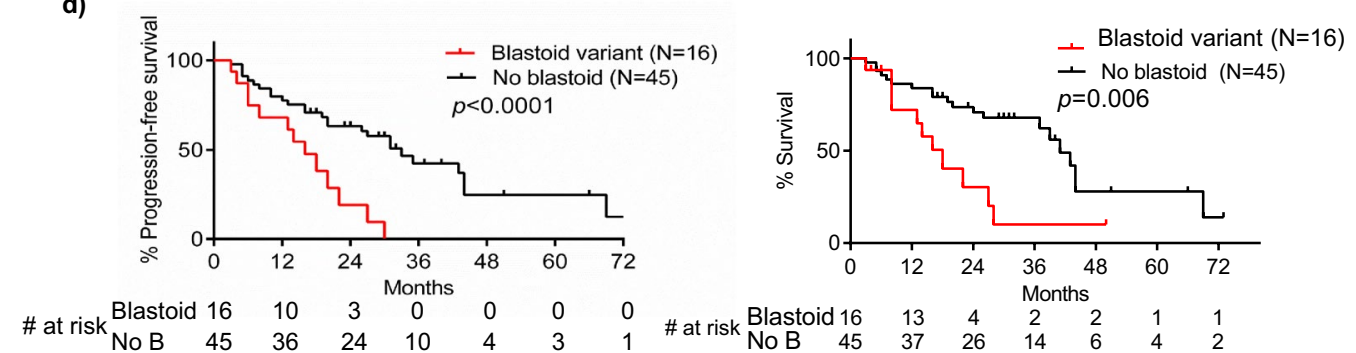

e)

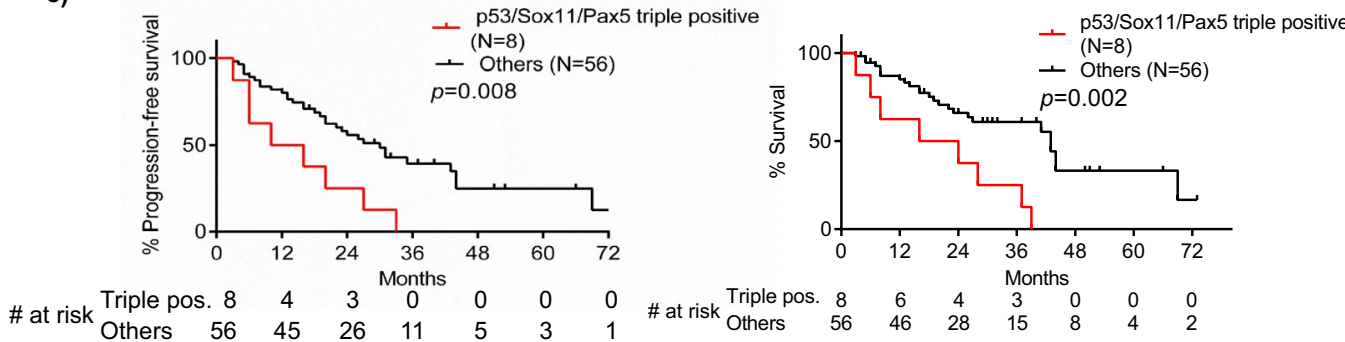

Figure 4. Risk factors of mantle cell lymphoma. (a) of 64 MCL patients, the median PFS was 14 versus 27 months (hazard ratio 2.657, $95 \%$ CI of ratio $1.595-12.78, p=0.002$ ) and the median OS was 14 versus 39 months (hazard ratio $2.663,95 \%$ CI of ratio $1.408-13.09$, $p=0.011$ ) for TP53 ${ }^{\text {mutt }}$ versus the others; (b) the median PFS was 20 versus 31 months (hazard ratio $1.791,95 \%$ CI of ratio $0.9221-$ $3.544, p=0.065$ ) and the median OS was 37 versus 43 months (hazard ratio $2.186,95 \%$ CI of ratio $1.014-4.739, p=0.049$ ) for MIPI $\geq 4$ patients versus the others; (c) the median PFS was 19 versus 44 months (hazard ratio $3.19,95 \% \mathrm{CI}$ of ratio $2.015-7.286, p<0.0001$ ) and the median OS was 20 versus 44 months (hazard ratio $2.934,95 \% \mathrm{CI}$ of ratio $1.545-6.441, p=0.002$ ) for Ki-67 ${ }^{\text {high }}$ patients versus the others; (d) the median PFS was 16 versus 33 months (hazard ratio $2.816,95 \% \mathrm{CI}$ of ratio $2.007-11.6, p<0.0001$ ) and the median OS was 18 versus 41 months (hazard ratio $2.616,95 \%$ CI of ratio $1.514-9.706, p=0.006$ ) for patients with blastoid variant cells versus the others. Three patients' blastoid variant information was not available. (e) The median PFS was 13 versus 30 months (hazard ratio 2.794, $95 \%$ CI of ratio $1.661-15.9, p=0.008$ ) and the median OS was 20 versus 43 months (hazard ratio $3.210,95 \%$ CI of ratio 2.067-22.26, $p=0.002)$ for triple-positive patients versus the others. 


\begin{tabular}{|c|c|c|c|}
\hline Variable & Triple positive & Others & $p$ value \\
\hline No. (\%) & $8(12.5)$ & $56(87.5)$ & \\
\hline Median age (range) & $62.5(47-72)$ & $61(36-82)$ & 0.911 \\
\hline Male, no. (\%) & $7(87.5)$ & $45(80.4)$ & $>0.99$ \\
\hline TP53 mutated, no. (\%) & $4(50)$ & $7(12.5)$ & 0.024 \\
\hline Median p53 H-score (range) & $89.55(42.38-204.56)$ & $34.5(8.89-214.61)$ & 0.001 \\
\hline Median Sox11 H-score (range) & $145.88(120.13-162.79)$ & $80.6(16.9-241.72)$ & 0.007 \\
\hline Median Pax5 H-score (range) & $160.84(114.4-250.36)$ & $84.12(28.84-258.86)$ & 0.012 \\
\hline $\mathrm{HB}, \mathrm{g} / \mathrm{L}($ mean $\pm \mathrm{SD})$ & $113.83 \pm 21.11$ & $123.82 \pm 24.14$ & 0.341 \\
\hline Median WBC, $10^{9} / \mathrm{L}$ (range) & $8.54(5.3-70.21)$ & $6.97(2.97-43.94)$ & 0.178 \\
\hline Median PLT, $10^{9} / \mathrm{L}$ (range) & $194(128-332)$ & $136(38-407)$ & 0.077 \\
\hline Median LDH, IU/L (range) & $328(151-827)$ & $218(136-424)$ & 0.515 \\
\hline MIPI ${ }^{\text {high }}$, no. (\%) & $2(28.6)$ & $12(24.5)$ & $>0.99$ \\
\hline Blastoid variant, no. (\%) & $3(37.5)$ & $12(22.6)$ & 0.393 \\
\hline Median Ki-67, \% (range) & $60(10-80)$ & $25(4-80)$ & 0.107 \\
\hline CD5-/ \pm , no. (\%) & $4(50)$ & $16(28.6)$ & 0.244 \\
\hline \multicolumn{3}{|l|}{ Treatment } & \multirow{4}{*}{0.845} \\
\hline CHOP (\%) & $3(37.5)$ & $15(26.8)$ & \\
\hline R-CHOP (\%) & $5(62.5)$ & $36(64.3)$ & \\
\hline R-CHOP/DHAP (\%) & $0(0)$ & $5(8.9)$ & \\
\hline
\end{tabular}

Table 3. Comparison of triple-positive patients with the other patients.

\begin{tabular}{|c|c|c|c|c|c|c|}
\hline \multirow[b]{2}{*}{ Variable } & \multicolumn{3}{|c|}{ Univariate } & \multicolumn{3}{|c|}{ Multivariate } \\
\hline & HR & 95\% CI & $p$ & HR & $95 \% \mathrm{CI}$ & $p$ \\
\hline \multicolumn{7}{|c|}{ Progression-free survival } \\
\hline Triple positive & 2.752 & $1.253-6.043$ & 0.012 & 2.476 & $0.985-6.224$ & 0.054 \\
\hline TP53 mut & 3.045 & $1.459-6.352$ & 0.003 & 1.821 & $0.759-4.368$ & 0.179 \\
\hline Ki- $67>30 \%$ & 3.253 & $1.662-6.366$ & 0.001 & 1.525 & $0.661-3.517$ & 0.322 \\
\hline MIPI (continuous) & 1.226 & $1.043-1.441$ & 0.014 & 1.107 & $0.915-1.339$ & 0.296 \\
\hline Blastoid variant & 3.839 & $1.842-8.001$ & $<0.0001$ & 2.962 & $1.249-7.023$ & 0.014 \\
\hline \multicolumn{7}{|l|}{ Overall survival } \\
\hline Triple positive & 3.387 & $1.481-7.745$ & 0.004 & 3.816 & $1.475-9.875$ & 0.006 \\
\hline TP53 mut & 2.729 & $1.210-6.152$ & 0.016 & 1.580 & $0.603-4.137$ & 0.352 \\
\hline Ki-67>30\% & 2.603 & $1.242-5.458$ & 0.011 & 1.085 & $0.42-2.805$ & 0.866 \\
\hline MIPI (continuous) & 1.255 & $1.041-1.513$ & 0.017 & 1.159 & $0.916-1.468$ & 0.219 \\
\hline Blastoid variant & 4.819 & $2.092-11.099$ & $<0.0001$ & 4.125 & $1.517-11.216$ & 0.005 \\
\hline
\end{tabular}

Table 4. Cox-regression analysis of triple-positive patients.

TP53 mutations and the patients' OS $^{7}$. Stefancikova and Zlamalikova et al. also were unable to find relevance between expression of p53 protein and MCL prognosis ${ }^{26,27}$. Thus, the single antigen expression of p53, Sox11, or Pax5 might not be sufficient to predict MCL prognosis. Of notice, most studies addressed the prognostic value of p53 expression in MCL using p53 protein instead of mRNA. P53 had post-transcriptional level regulations, such as translation regulation by internal ribosomal entry site (IRES) ${ }^{28}$ and protein stability regulation ${ }^{29}$. Thus, there might be inconsistence between p53 expressions at the mRNA level and the protein level.

Our result suggested that the MCL patients with triple-positive expression of p53/Sox11/Pax 5 had inferior OS. Such correlation did not stand on any identified prognostic factors of MCL, such as MIPI, Ki-67, or TP53 mutation status. Why triple-positive MCL had inferior OS were not clear. Large cohort study was necessary to draw a confirmative conclusion. Previous studies demonstrated that Sox 11 played an oncogenic role in MCL by modulating cell cycle, apoptosis, and cell differentiation. Pax 5 was regulated by Sox 11 at the transcriptional level ${ }^{11}$. On the other hand, the function of p53 in MCL, in particular its interplay with other MCL oncogenic factors, was not well demonstrated. Yang P et al. suggested that aberrant p53 might promote cell cycle dysregulation in $\mathrm{MCL}^{30}$. Teo et al. mentioned that the TP53-/- MCL cell line had altered chemo-sensitivity ${ }^{22}$. Based on the above findings, overexpression of $\mathrm{p} 53$, Sox11, or Pax5 promotes MCL malignancy. Thus, it was reasonable to predict that $\mathrm{p} 53 /$ Sox11/Pax 5 triple-positive MCL might represent a highly aggressive tumor subtype with inferior prognosis. In our data, the inconsistence of Sox 11 and Pax 5 co-expression in MCL suggested that, at least in some MCL, the Pax 5 expression might be controlled by factors other than Sox11. Furthermore, Teo et al. showed that Pax-/- MCL had increased cell proliferation in vitro and more aggressive tumor behavior in vivo ${ }^{22}$. Teo's study 
also indicated a Pax5-initiated regulation on p53. Therefore, in some MCL, Sox11 and Pax5 regulatory hierarchy and $\mathrm{p} 53$ regulation might not be canonical as we had anticipated.

It should be noted that there are several limitations of our study. (1) Small patient number; (2) heterogeneous treatment regimen; (3) lacks the study of mechanisms. Therefore, additional prospective studies with lager cohorts and homogenous treatment regimen are needed to verify our results. The potential pathophysiological mechanisms of these results deserve further investigation.

To summarize, the MCL patients with triple positive p53/Sox11/Pax5 expression had inferior disease. Such MCL risk stratification was independent to MIPI, Ki-67, or TP53 mutation status. The biology underneath such observation is still largely unknown. Future validation and demonstration of this MCL risk stratification might provide new perspective on MCL pathology.

\section{Materials and methods}

Patient samples. Formalin-fixed, paraffin-embedded tissue biopsies from 64 MCL patients were included in this study. The patients were diagnosed in West China Hospital, Sichuan University from 2012 to 2016. The MCL diagnostic standards were based on WHO guidelines ${ }^{31}$. All patients had cyclin D1 overexpression in tissue biopsies and had $\mathrm{t}(11 ; 14)(\mathrm{q} 13 ; \mathrm{q} 32)$ detected by fluorescence in situ hybridization (FISH). The patients received R-CHOP/CHOP (Rituximab, Cyclophosphamide, Doxorubicin, Oncovin, Prednisone), or R-DHAP (Dexamethasone, Cytarabine, Cisplatin) treatment, Among them, two patients received rituximab maintenance, one patient received autologous stem cell transplantation (ASCT). Of the 64 MCL patients, the median follow-up time was 23 months with a range of 3-73 months. The Ethical Committee of Sichuan University approved this study and waived informed consent. All methods were performed in accordance with the relevant guidelines and regulations.

Assessment of TP53 mutations in patient samples. Genomic DNA was isolated from paraffinembedded tissues using QIAmp DNA FFPE Tissue Kit (Qiagen) following the manufacturer's instruction. TP53 exons 2-11, including the intron-exon boundaries, were sequenced by Sanger sequencing. Supplementary Table S4 given the primer sequences. The result was compared with the International Agency for Research on Cancer (IARC) TP53 database, the Catalogue of Somatic Mutations in Cancer (COSMIC) database, and the UMD TP53 mutation database to identify novel mutations. Wild-type TP53 sequence was acquired from NCBI.

Immunohistochemical analysis of tissue array and results quantification. Paraffin-embedded tissue biopsies from the patients were sectionized and used for IHC analysis as described earlier ${ }^{32}$. The primary antibody against human p53 was ordered from Thermo Fisher Inc (MA5-12557). The primary antibody against Sox11 was ordered from ZSBIO (ZM-0366), CHINA. The primary antibodies against Pax5 was ordered from Abcam (ab109443). All antibodies were diluted with phosphate buffer solution (PH 7.3) and had been verified for immunohistochemistry staining. Percentage of MCL cells in the biopsy samples and positive nuclei in the MCL cells were determined by 2 pathologists under microscopy independently. The results were in good concordance. For the different results, 2 pathologists determined the end results together. According to a previous study ${ }^{15}$ and appropriate adjustments, proteins were defined as negative ( $0 \%$ positive MCL cells), low (1-29\% positive MCL cells), intermediate (30-49\% positive MCL cells) or high ( $\geq 50 \%$ positive MCL cells). The results of staining were captured by microscopy using AX10 Imager A1/Cam HRC and Zeiss at $\times 40$ magnification, and were quantified by ImageJ with the IHC profiler plugin as described earlier ${ }^{33}$. The results were rendered as a pixel intensity histogram to demonstrate the percentage contribution of high positive, positive, low positive, and negative. The scoring assignment was performed via $\mathrm{H}$-scoring. The formula was as follows: $\mathrm{H}$-score $=(\%$ of high positive $\times 3)+(\%$ of positive $\times 2)+(\%$ of low positive $\times 1)$. The $\mathrm{H}$-score had a range from 0 to 300 . Based on a previous publication ${ }^{34}$, with some modifications, we defined $\mathrm{H}$-score $>100$ as positive for Sox 11 and Pax 5 and $\mathrm{H}$-score $>40$ as positive for $\mathrm{p} 53$.

Statistical analysis. Statistical analyses were performed using SPSS Statistics (Version 19) and GraphPad. Comparisons of two groups were analyzed by Student's $t$-test. Specifically, the Mann-Whitney test was used for quantitative variables and $\chi^{2}$ or Fisher's exact test was used for categorical variables. The receiver operating characteristic (ROC) curve was used to analyze the level of p53 IHC expression in predicting TP53 mutation. Progression-free survival (PFS) and overall survival (OS) were estimated and compared using the Kaplan-Meier method (Log-rank test). Cox proportional hazard models (univariate and multivariate) were used to identify independent prognostic factors. A $p<0.05$ was considered statistically significant.

\section{Data availability}

The authors declare that the main data supporting the results of the current study are available from the corresponding authors on reasonable request.

Received: 31 May 2020; Accepted: 24 May 2021

Published online: 07 June 2021

\section{References}

1. Skarbnik, A. P. \& Goy, A. H. Mantle cell lymphoma: State of the art. Clin. Adv. Hematol. Oncol. 13, 44-55 (2015).

2. Aschebrook-Kilfoy, B., Caces, D. B., Ollberding, N. J., Smith, S. M. \& Chiu, B. C. An upward trend in the age-specific incidence patterns for mantle cell lymphoma in the USA. Leuk. Lymphoma 54, 1677-1683. https://doi.org/10.3109/10428194.2012.760041 (2013). 
3. Jares, P., Colomer, D. \& Campo, E. Molecular pathogenesis of mantle cell lymphoma. J. Clin. Investig. 122, 3416-3423. https://doi. org/10.1172/JCI61272 (2012).

4. Pinyol, M. et al. p16 (INK4a) gene inactivation by deletions, mutations, and hypermethylation is associated with transformed and aggressive variants of non-Hodgkin's lymphomas. Blood 91, 2977-2984 (1998).

5. Schaffner, C., Idler, I., Stilgenbauer, S., Dohner, H. \& Lichter, P. Mantle cell lymphoma is characterized by inactivation of the ATM gene. Proc. Natl. Acad. Sci. U.S.A. 97, 2773-2778. https://doi.org/10.1073/pnas.050400997 (2000).

6. Hernandez, L. et al. CDK4 and MDM2 gene alterations mainly occur in highly proliferative and aggressive mantle cell lymphomas with wild-type INK4a/ARF locus. Cancer Res. 65, 2199-2206. https://doi.org/10.1158/0008-5472.CAN-04-1526 (2005).

7. Greiner, T. C. et al. P53 mutations in mantle cell lymphoma are associated with variant cytology and predict a poor prognosis. Blood 87, 4302-4310 (1996).

8. Salaverria, I. et al. Specific secondary genetic alterations in mantle cell lymphoma provide prognostic information independent of the gene expression-based proliferation signature. J. Clin. Oncol. 25, 1216-1222 (2007).

9. Fernandez, V. et al. Genomic and gene expression profiling defines indolent forms of mantle cell lymphoma. Cancer Res. 70, 1408-1418. https://doi.org/10.1158/0008-5472.CAN-09-3419 (2010).

10. Vose, J. M. et al. Mantle cell lymphoma: 2017 update on diagnosis, risk-stratification, and clinical management. Am. J. Hematol. 92, 806-813. https://doi.org/10.1002/ajh.24797 (2017).

11. Vegliante, M. C. et al. SOX11 regulates PAX5 expression and blocks terminal B-cell differentiation in aggressive mantle cell lymphoma. Blood 121, 2175-2185. https://doi.org/10.1182/blood-2012-06-438937 (2013).

12. Patocs, A. et al. Breast-cancer stromal cells with TP53 mutations and nodal metastases. N. Engl. J. Med. 357, 2543-2551. https:// doi.org/10.1056/NEJMoa071825 (2007).

13. Cheung, K. J., Horsman, D. E. \& Gascoyne, R. D. The significance of TP53 in lymphoid malignancies: Mutation prevalence, regulation, prognostic impact and potential as a therapeutic target. Br. J. Haematol. 146, 257-269. https://doi.org/10.1111/j.1365-2141. 2009.07739.x (2009).

14. Nygren, L. et al. Prognostic role of SOX11 in a population-based cohort of mantle cell lymphoma. Blood 119, 4215-4223. https:// doi.org/10.1182/blood-2011-12-400580 (2012).

15. Aukema, S. M. et al. Expression of TP53 is associated with the outcome of MCL independent of MIPI and Ki-67 in trials of the European MCL Network. Blood 131, 417-420. https://doi.org/10.1182/blood-2017-07-797019 (2018).

16. Hoster, E. et al. Prognostic value of Ki-67 index, cytology, and growth pattern in mantle cell lymphoma: Results from randomized trials of the European mantle cell lymphoma network. J. Clin. Oncol. 34, 1386-1394. https://doi.org/10.1200/JCO.2015.63.8387 (2016).

17. Ek, S., Dictor, M., Jerkeman, M., Jirstrom, K. \& Borrebaeck, C. A. Nuclear expression of the non B-cell lineage Sox11 transcription factor identifies mantle cell lymphoma. Blood 111, 800-805. https://doi.org/10.1182/blood-2007-06-093401 (2008).

18. Choe, J. Y. et al. MYC overexpression correlates with MYC amplification or translocation, and is associated with poor prognosis in mantle cell lymphoma. Histopathology 68, 442-449. https://doi.org/10.1111/his.12760 (2016).

19. Halldorsdottir, A. M. et al. Impact of TP53 mutation and 17p deletion in mantle cell lymphoma. Leukemia 25, 1904-1908. https:// doi.org/10.1038/leu.2011.162 (2011).

20. Wang, Y. \& Ma, S. Risk factors for etiology and prognosis of mantle cell lymphoma. Expert Rev. Hematol. 7, 233-243. https://doi. org/10.1586/17474086.2014.889561 (2014).

21. Dong, H. J. et al. TP53 mutation is not an independent prognostic factor in patients with mantle cell lymphoma at advanced stage. Med. Oncol. 29, 2166-2173. https://doi.org/10.1007/s12032-011-0096-5 (2012).

22. Teo, A. E. et al. Differential PAX5 levels promote malignant B-cell infiltration, progression and drug resistance, and predict a poor prognosis in MCL patients independent of CCND1. Leukemia 30, 580-593. https://doi.org/10.1038/leu.2015.140 (2016).

23. Swerdlow, S. H. et al. WHO Classification of Tumours of Haematopoietic and Lymphoid Tissues 285-290 (International Agency for Research on Cancer, 2017).

24. Navarro, A. et al. Molecular subsets of mantle cell lymphoma defined by the IGHV mutational status and SOX11 expression have distinct biologic and clinical features. Cancer Res. 72, 5307-5316. https://doi.org/10.1158/0008-5472.CAN-12-1615 (2012).

25. Nordstrom, L. et al. SOX11 and TP53 add prognostic information to MIPI in a homogenously treated cohort of mantle cell lymphoma-A nordic lymphoma group study. Br. J. Haematol. 166, 98-108. https://doi.org/10.1111/bjh.12854 (2014).

26. Stefancikova, L. et al. Loss of the p53 tumor suppressor activity is associated with negative prognosis of mantle cell lymphoma. Int. J. Oncol. 36, 699-706. https://doi.org/10.3892/ijo_00000545 (2010).

27. Zlamalikova, L. et al. Complex analysis of the TP53 tumor suppressor in mantle cell and diffuse large B-cell lymphomas. Oncol. Rep. 38, 2535-2542. https://doi.org/10.3892/or.2017.5891 (2017).

28. Yang, D. Q., Halaby, M. J. \& Zhang, Y. The identification of an internal ribosomal entry site in the 5'-untranslated region of p53 mRNA provides a novel mechanism for the regulation of its translation following DNA damage. Oncogene 25, 4613-4619. https:// doi.org/10.1038/sj.onc.1209483 (2006).

29. Pan, M. \& Blattner, C. Regulation of p53 by E3s. Cancers (Basel). https://doi.org/10.3390/cancers13040745 (2021).

30. Yang, P. et al. Genomic landscape and prognostic analysis of mantle cell lymphoma. Cancer Gene Ther. 25, 129-140. https://doi. org/10.1038/s41417-018-0022-5 (2018).

31. Sabattini, E., Bacci, F., Sagramoso, C. \& Pileri, S. A. WHO classification of tumours of haematopoietic and lymphoid tissues in 2008: An overview. Pathologica 102, 83-87 (2010).

32. Hong, B. et al. P38 MAPK inhibits breast cancer metastasis through regulation of stromal expansion. Int. J. Cancer 136, 34-43. https://doi.org/10.1002/ijc.28958 (2015).

33. Varghese, F., Bukhari, A. B., Malhotra, R. \& De, A. IHC Profiler: An open source plugin for the quantitative evaluation and automated scoring of immunohistochemistry images of human tissue samples. PLoS ONE 9, e96801. https://doi.org/10.1371/journal. pone.0096801 (2014).

34. Bydoun, M. et al. S100A10, a novel biomarker in pancreatic ductal adenocarcinoma. Mol. Oncol. 12, 1895-1916. https://doi.org/ 10.1002/1878-0261.12356 (2018).

\section{Acknowledgements}

The author is grateful to the Pathology Department of West China Hospital of Sichuan University for their valuable assistance during this study. This work was supported by Department of Science and Technology of Sichuan Province for key research and development projects under Grant 2019YFS0027.

\section{Author contributions}

C.X. initiated the project, designed the research. C.J. performed majority of the experiments, analyzed the data, and prepared the manuscript. Y.Z. designed the research, analyzed the data and prepared the manuscript. Y.F. and X.C. performed experiments and provided critical suggestions. All authors concur with the submission and the content of the manuscript. 


\section{Competing interests}

The authors declare no competing interests.

\section{Additional information}

Supplementary Information The online version contains supplementary material available at https://doi.org/ 10.1038/s41598-021-91433-7.

Correspondence and requests for materials should be addressed to C.X.

Reprints and permissions information is available at www.nature.com/reprints.

Publisher's note Springer Nature remains neutral with regard to jurisdictional claims in published maps and institutional affiliations.

(c) (1) Open Access This article is licensed under a Creative Commons Attribution 4.0 International License, which permits use, sharing, adaptation, distribution and reproduction in any medium or format, as long as you give appropriate credit to the original author(s) and the source, provide a link to the Creative Commons licence, and indicate if changes were made. The images or other third party material in this article are included in the article's Creative Commons licence, unless indicated otherwise in a credit line to the material. If material is not included in the article's Creative Commons licence and your intended use is not permitted by statutory regulation or exceeds the permitted use, you will need to obtain permission directly from the copyright holder. To view a copy of this licence, visit http://creativecommons.org/licenses/by/4.0/.

(C) The Author(s) 2021 